\title{
Neuroimaging in Neuro-Oncology
}

\author{
Soonmee Cha \\ Department of Radiology, University of California, San Francisco Medical Center, San Francisco, California 94143-0628
}

\begin{abstract}
Summary: Neuroimaging of brain tumors has evolved from a strictly morphology-based discipline to one that encompasses function, physiology, and anatomy. This review outlines the current imaging standard for patients with brain tumor and summarizes the latest advances in physiology-based imaging methods that complement traditional brain tumor imaging protocols. Emphasis is on the strength and limitations of the current imaging standards and on an overview of several advanced imaging meth-
\end{abstract}

ods including diffusion-weighted magnetic resonance imaging (MRI), perfusion MRI, and proton magnetic resonance spectroscopic (MRS) imaging. Basic physical principles behind each imaging method are briefly presented, along with a more in-depth discussion of clinical application and potential pitfalls of each technique. Key Words: Brain tumor, brain cancer, glioma, glioblastoma multiforme, radiation necrosis, MRI, diffusionweighted MRI, perfusion MRI, proton spectroscopy.

\section{INTRODUCTION}

Brain tumors include a variety of subtypes with a wide range of histopathology, molecular and genetic profile, clinical spectrum, treatment possibilities, and patient prognosis and outcome. The complex pathophysiology of brain tumor is dependent on various factors, including histology, molecular and chromosomal aberration, tumor-related protein expression, primary versus secondary origin, and host factors. ${ }^{1-4}$

Compared with systemic cancers (e.g., lung, breast, prostate, colon), brain tumor is unique in several different ways. First, the brain is covered by a tough, fibrous tissue dura mater and a bony skull that protects the inner contents. This rigid covering allows very little, if any, increase in volume of the inner content. Brain tumor cells, therefore, adapt to grow more in an infiltrative than an expansive pattern. This growth pattern limits the disruption to the underlying cytoarchitecture. Second, brain capillaries have a unique barrier known as the bloodbrain barrier (BBB), which limits the entrance of systemic circulation into the central nervous system. Cancer cells can hide behind the protective barrier of the BBB, migrate with minimal disruption to the structural and physiologic milieu of the brain, and escape imaging de-

Address correspondence and reprint requests to: Soonmee Cha, M.D., Department of Radiology, University of California, San Francisco Medical Center, 505 Parnassus Avenue, Box 0628, Room L358, San Francisco, CA 94143-0628. E-mail: soonmee.cha@radiology. ucsf.edu. tection because intravenous contrast agent becomes visible when there is BBB disruption, allowing the agent to leak into the interstitial space.

Neuroimaging plays a critical role in the diagnosis and preoperative planning of brain tumor cases and also serves as a means for evaluation during or after therapy. The type of imaging acquired depends on the particular clinical setting, the time point, and the information the clinician needs to make treatment decisions. Neuroimaging of brain tumor can be divided into three broad categories, serving three specific clinical questions: 1) diagnosis, 2) preoperative or therapy planning, and 3) posttreatment evaluation. Once the subject is identified as high-risk for suspected brain tumor, available evidence suggests that magnetic resonance imaging (MRI) with and without gadolinium-based contrast agent is the recommended imaging test of choice. The true costeffectiveness of MRI for adult subjects in this clinical scenario, however, has not been performed. Computed tomography scanners are more widely available than MRI scanners, and the scans are more easily performed, especially in an emergency department setting. Because of this, CT is commonly performed even though it is inferior to MR in tumor detection and characterization. There is no evidence that supports the notion that the combination of CT and MRI either improves the outcome or the cost-effectiveness for patients with brain tumor.

Recent advances in technology have fostered the development and clinical application of several new phys- 
iology-based MRI methods that provide information that is not readily available from traditional anatomical imaging. These methods include diffusion-weighted MRI, contrast-enhanced perfusion MRI, and proton MRS imaging. There are exciting and important advances in imaging methods other than in the field of MRI, most notably in the discipline of molecular imaging, but this topic is well beyond the scope of the present review. Instead, the focus here is on discussion of current MRI standards for diagnosis of brain tumor and an overview of new physiology-based MRI methods and their potential roles in improving tumor characterization and understanding tumor biology.

\section{NEUROIMAGING OF BRAIN TUMORS}

Unenhanced CT of the brain is considered the first line of imaging to evaluate patients with suspected brain tumor who present with acute symptoms. Computed tomography is widely available, fast and easy to perform, relatively risk-free, and well tolerated; it is very sensitive in detecting acute hemorrhage, hydrocephalus, and herniation. Contrast-enhanced CT can be helpful in detecting areas of BBB and defining the contrast-enhancing tumor border. Despite its many advantages, however, CT suffers from several important limitations when it comes to brain tumor imaging. First, CT is not optimally suited for detecting subtle changes in brain parenchyma, such as nonenhancing tumor or infiltrative changes, because of its intrinsically low soft tissue contrast. ${ }^{5}$ The CT findings can be subtle in cases of infiltrating tumor and easily overlooked (FIG. 1). Second, CT does not provide flexible multiplanar acquisition, which limits the three-dimensional depiction of tumor; CT is largely confined to providing anatomic information. Even with the addition of intravenous contrast agent, CT is still inferior to MRI in terms of soft tissue resolution, multiplanar capability, and physiology-based applications. Finally, CT involves ionizing radiation and its iodinated contrast agent can cause serious allergic reaction. For these reasons, CT is primarily reserved as an initial screening method to exclude potentially life-threatening intracranial process.

Magnetic resonance imaging is the imaging test of choice for patients with brain tumor before or after therapy. Intravenous MRI contrast agent, gadolinium, is a requisite in brain tumor imaging. Unlike the iodinated contrast agent used for $\mathrm{CT}$, a gadolinium-based contrast agent is better tolerated and has a much lower risk profile. Contrast-enhanced MRI provides exquisite anatomical detail, has multiplanar capability, and does not require ionizing radiation; it is therefore the current imaging standard for brain tumor diagnosis and therapy monitoring. In clinical practice, there are numerous different MRI protocols for imaging brain tumor. The most widely accepted standard imaging protocol includes at least the following three sequences: contrast-enhanced $\mathrm{T}_{1}$-weighted imaging, fluid-attenuated inversion recovery (FLAIR), and diffusion-weighted imaging (DWI) (FIG. 2).

Contrast-enhanced $\mathrm{T}_{1}$-weighted imaging is among the most important MRI sequences for characterization of brain tumor. ${ }^{6}$ The areas of contrast enhancement on $\mathrm{T}_{1^{-}}$weighted images represent regions of BBB breakdown, in which gadolinium has leaked out. This is a process more commonly seen with aggressive neoplasms, such as high-grade glioma. Contrast-enhancing tumor is not, however, synonymous with a malignant brain tumor. Indolent or less aggressive tumors, such as pilocytic astrocytomas or meningiomas, often demonstrate contrast enhancement. Determination of tumor enhancement must be done by evaluating both nonenhanced and enhanced $\mathrm{T}_{1}$-weighted images, so as not to confuse intrin-
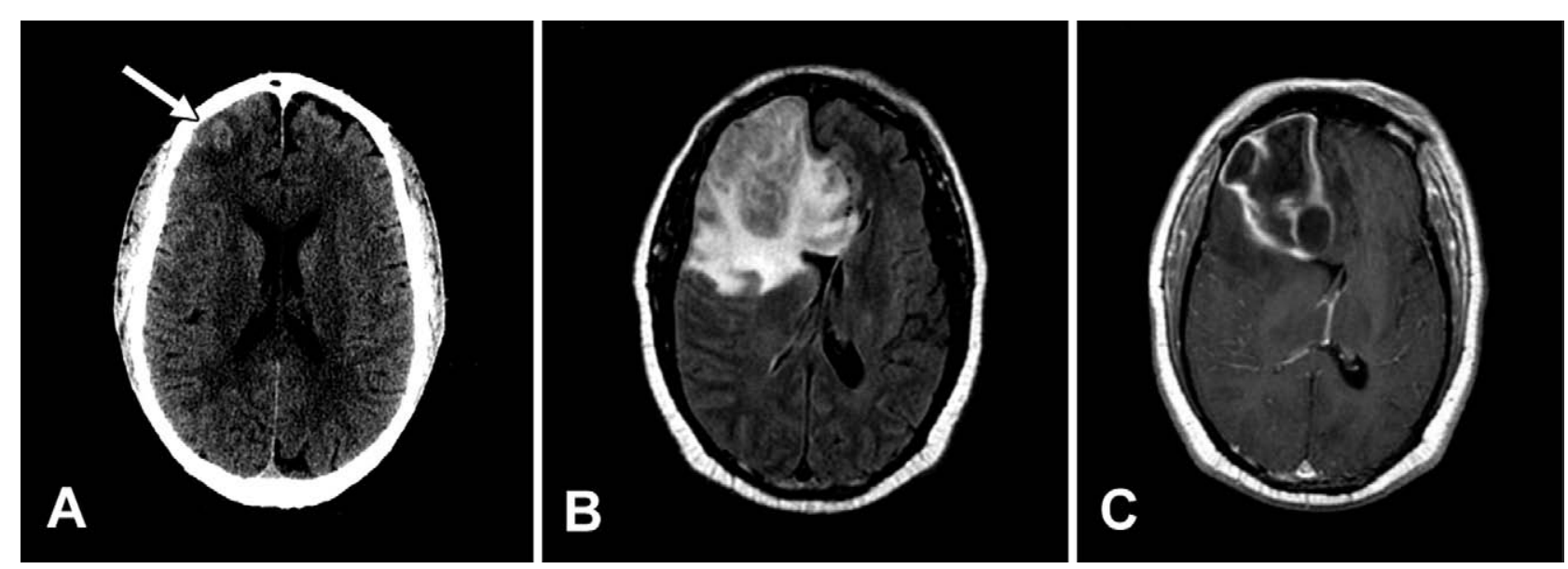

FIG. 1. A 41-year-old man with right frontal pyogenic cerebral abscess. A: Unenhanced axial CT image of the brain shows a subtle lesion in the right frontal lobe (arrow). B: Axial fluid-attenuated inversion recovery (FLAIR) MR image done 1 day after the CT shows a large mass lesion in the right frontal lobe. $\mathrm{C}$ : Axial contrast-enhanced $\mathrm{T}_{1}$-weighted image clearly delineates the margins of the right frontal mass lesion. 

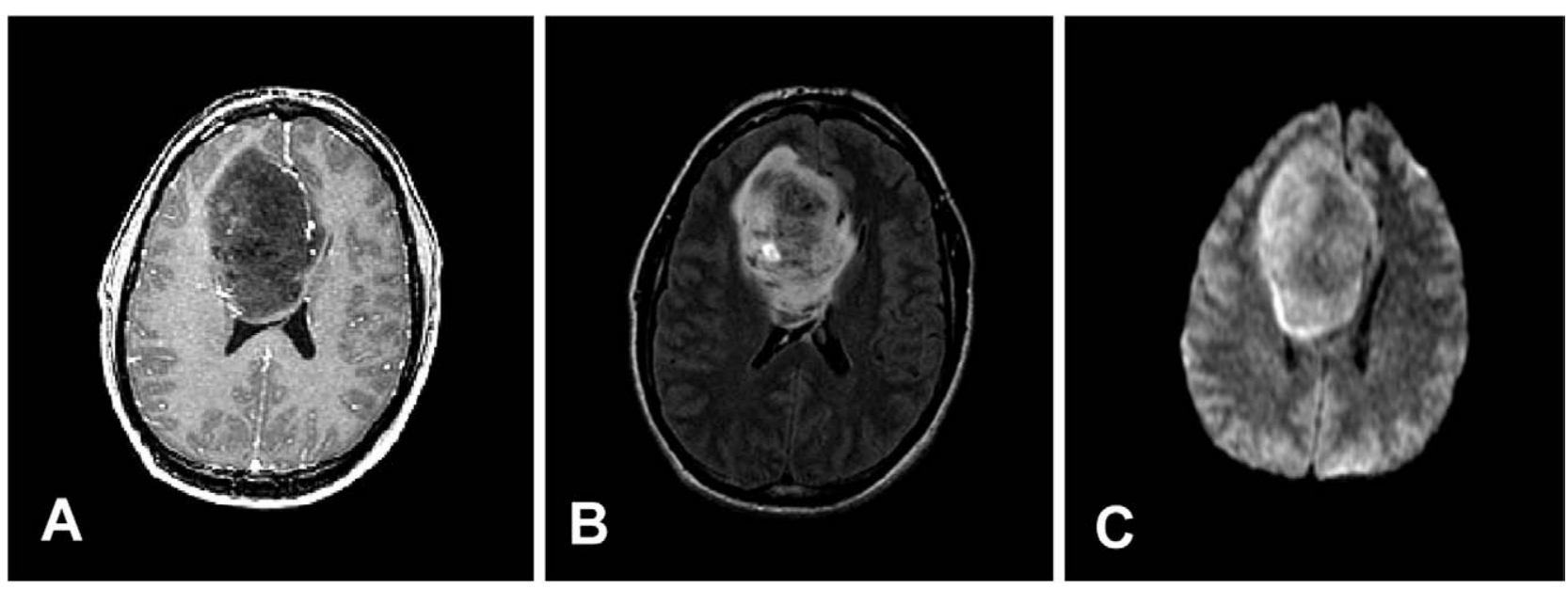

FIG. 2. A 28-year-old man with right frontal anaplastic astrocytoma. A: Axial contrast-enhanced $T_{1}$-weighted image shows a mostly nonenhancing large right frontal lobe mass causing mass effect on the lateral ventricle. B: Axial FLAIR image demonstrates heterogeneous signal characteristics within the mass. C: Axial diffusion-weighted image shows similar heterogeneity of the mass.

sically $\mathrm{T}_{1}$ bright lesions (usually those containing blood products, fat, or proteinaceous material) with enhancing ones (FIG. 3).

Brain tumor imaging involves characterization not only of the core tumor, but also of reactive and infiltrative changes surrounding the tumor. Tumor-related edema can be subtle on contrast-enhanced $\mathrm{T}_{1}$-weighted images, but is readily apparent on $\mathrm{T}_{2}$-weighted images because of to their higher sensitivity to changes in water content of the brain. Fluid-attenuated inversion recovery (FLAIR) imaging is a type of $\mathrm{T}_{2}$-weighted imaging in which the signal from CSF is suppressed in order to increase the conspicuity of lesions adjacent to ventricles or sulci. ${ }^{7}$ FLAIR imaging is very sensitive to subtle differences in soft tissue contrast and can depict the full extent of tumor and surrounding tumor-related changes far better than contrast-enhanced $\mathrm{T}_{1}$-weighted imaging (FIG. 2). In addition, FLAIR imaging has been shown to be superior to contrast-enhanced $\mathrm{T}_{1}$-weighted imaging in detecting subtle leptomeningeal spread of tumor, as well as in demonstrating low-contrast lesions and subarachnoid hemorrhage. ${ }^{8,9}$

Despite its superb soft tissue contrast, multiplanar capability, and noninvasive nature, anatomical MRI, as described above, is largely limited to depicting morphological abnormality. In addition, anatomical MRI suffers from nonspecificity. Different disease processes can appear similar upon anatomic imaging, and in turn a single disease entity may have varied imaging findings. The underlying metabolic or functional integrity of brain cannot be adequately evaluated based on anatomical MRI alone. To that end, several physiology-based MRI methods have been developed to improve tumor characterization.

Among many advances in MRI methods, three are discussed here: diffusion-weighted MRI, contrast-en-
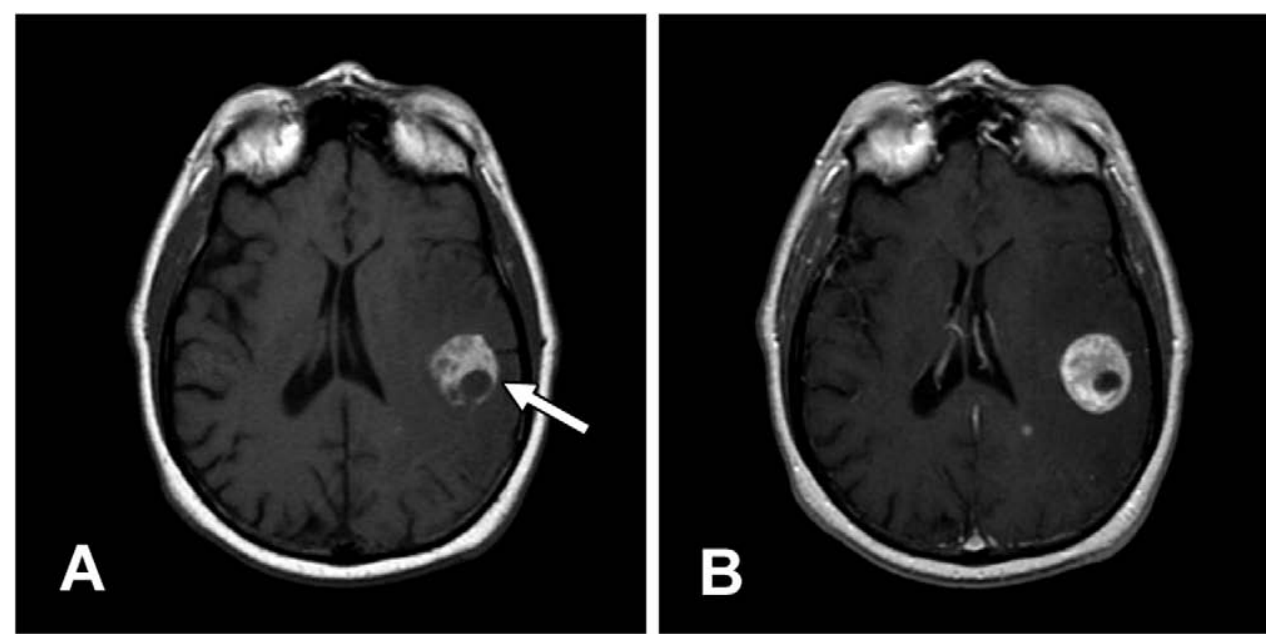

FIG. 3. A 55-year-old man with left parietal metastatic brain cancer from lung primary cancer. A: Axial precontrast $T_{1}$-weighted image shows a bright lesion in the left temporoparietal lobe, consistent with underlying blood products. B: Axial postcontrast $T_{1}$-weighted image shows some enhancement of the dominant mass, but also demonstrates a smaller lesion medially. 
hanced perfusion MRI, and proton MRS imaging. Most of these advanced MRI methods are still considered investigational and require further clinical validation and outcomes data. Nonetheless, physiology-based MRI methods are rapidly becoming an integral part of routine brain tumor imaging protocols and it is therefore important to understand the basic principles of each technique, along with the strengths and potential pitfalls.

\section{PHYSIOLOGY-BASED MRI}

\section{Diffusion-weighted MRI}

Diffusion is defined as the process of random molecular thermal motion occurring at a microscopic scale. Diffusion of water in biologic systems, particularly within the brain, is affected not only by the complex interaction between the intracellular and extracellular compartments, but also by the cytoarchitecture of the microstructures and permeability barriers. Diffusion of water molecules through the magnetic field gradient produces intravoxel dephasing and a loss of signal intensity. Because this microscopic diffusional motion is so small, a large gradient strength and/or duration is needed to produce observable signal loss from diffusion. With the use of bipolar pulsed gradient methods, microscopic diffusional motion is detected in terms of change in the magnitude of moving spins due to phase dispersion. To detect this highly sensitive motion, an ultrafast imaging technique (such as the echo-planar technique) is needed, one that can acquire a sufficient number of images (over a scan period in the range of milliseconds) to produce meaningful information.

The apparent diffusion coefficient (ADC) characterizes the rate of diffusional motion (in millimeters squared per second). The ADC takes into consideration the heterogeneous environment of brain cytoarchitecture and also factors other than diffusion, such as temperature, perfusion, and metabolic rates that can affect the measurement of microscopic thermal motion. High ADC implies relatively unrestricted water motion. Low ADC indicates restricted diffusional motion, as seen in acute cerebral ischemia. The diffusion sensitivity parameter, the $b$-value, is related to duration, strength, and time interval between the diffusion-sensitizing gradients. A typical $b$-value used in clinical imaging is in the range of 900 to 1000 $\mathrm{s} / \mathrm{mm}^{2}$. The higher the $b$-value, the more sensitive the diffusion imaging is in obtaining greater contrast and detecting areas of restricted water motion.

Echo-planar imaging ${ }^{10}$ is currently the most widely used MRI technique in clinical application of diffusionweighted imaging for the diagnosis of acute stroke and other brain disorders, such as abscess, epidermoid, traumatic shearing injury, or necrotic encephalitis. Echoplanar imaging, the fastest available MRI method, allows the entire set of echoes needed to form an image to be collected within a single acquisition period of 25 to $100 \mathrm{~ms} .^{11}$ The data are obtained through forming a train of gradient echoes by repeated reversal of a large gradient capable of very rapid polarity inversion to complete $k$-space filling after a single radiofrequency (RF) pulse. Each gradient echo is phase-encoded separately by a very brief blipped gradient or a weak constant phaseencoding gradient. Although the long echo train renders the images sensitive to chemical shift and to magnetic susceptibility artifacts, echo-planar imaging virtually eliminates motion artifact. The chemical-shift artifact is overcome by routine use of lipid suppression whereas the magnetic susceptibility artifact is manifested prominently at air-bone-tissue interfaces such as those at the skull base, paranasal sinuses, orbits, and petrous temporal bone. ${ }^{12-14}$

Diffusion-tensor magnetic resonance imaging and fiber tractography are new diffusion-weighted imaging methods that can demonstrate the orientation and integrity of white matter fibers in vivo. Although they remain investigational at this time, both diffusion-tensor magnetic resonance imaging and fiber tractography show much promise in assessing the integrity of white matter tracts and promise to provide much needed information for preoperative planning for brain tumors in and around eloquent white matter tracts. ${ }^{15-18}$

\section{Clinical application of diffusion-weighted MRI}

In addition to early diagnosis of cerebral ischemia, diffusion-weighted MRI is extremely sensitive in detecting other intracranial disease processes, including cerebral abscess, epidermoid, traumatic shearing injury, toxic or infectious encephalitis, and immediate postoperative brain injury. The exact mechanism of diffusion restriction in each of these disease entities is not yet entirely clear, but diffusion-weighted MRI can be extremely helpful in making the diagnosis.

Cerebral abscess, for example, can appear indistinguishable from cystic brain tumor, and preoperative diagnosis is critical for proper surgical and medical management. Diffusion-weighted MRI demonstrates profound restricted diffusion abnormality in cerebral abscess (FIG. 4), most likely attributable to the increased viscosity of the pus fluid. Similarly, the contents of epidermoid cyst contain highly viscous material, which can explain the characteristic restricted diffusion associated with this entity (FIG. 5). Diffuse axonal injury after trauma represents areas of permanent brain injury due to rotational shearing force, which results in destruction of cytoarchitecture of the affected brain. In toxic or infectious encephalitis (herpes encephalitis being the prototype), there is widespread necrotizing tissue destruction due to direct injury to the neurons and oligodendrocytes. Again, the end result is altered membrane integrity and shift of water 

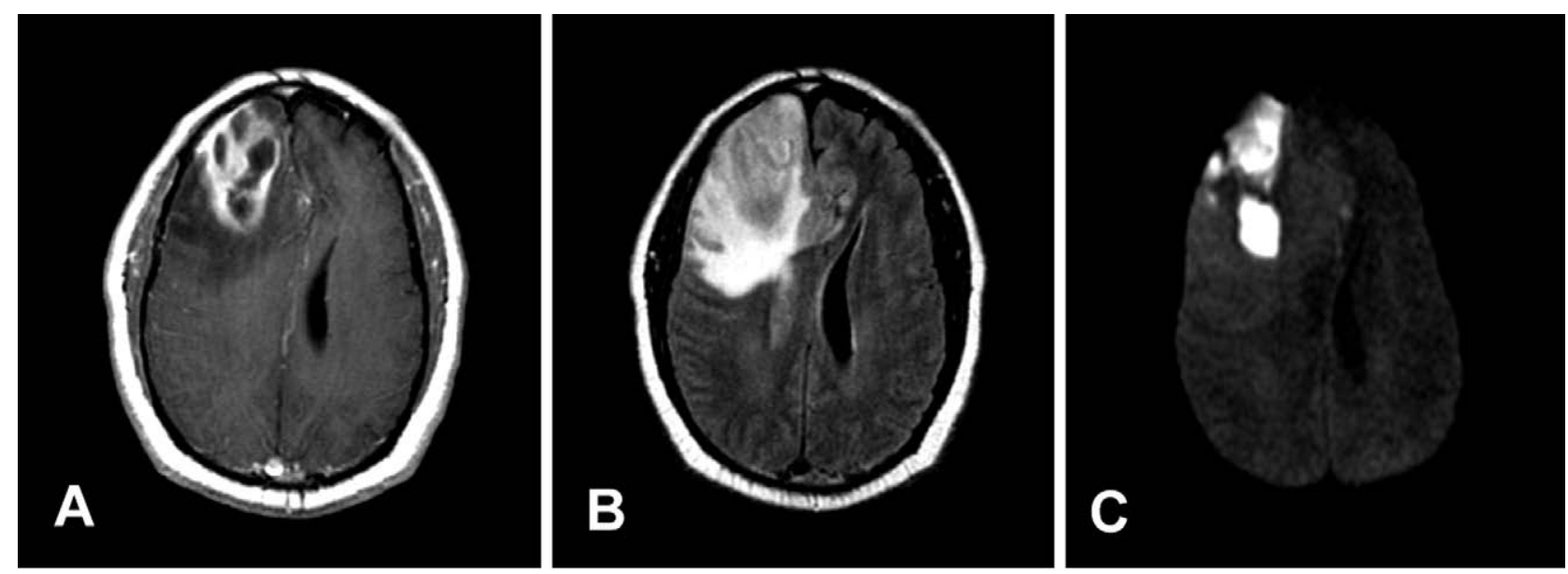

FIG. 4. A 41-year-old man with right frontal pyogenic cerebral abscess (same patient as FIG. 1). A: Axial contrast-enhanced $T_{1}$ weighted image shows an aggressive-appearing right frontal lobe mass. B: Axial FLAIR image demonstrates extensive edema surrounding the mass. C: Axial diffusion-weighted image shows marked restricted diffusion (bright signal) within the mass, consistent with a pyogenic abscess, which was confirmed at surgery.

into the intracellular compartment and corresponding restriction of extracellular space and water motion.

Diffusion-weighted MRI can be extremely valuable in accurate interpretation of new abnormal contrast enhancement that develops soon after tumor resection. In FIG. 6, an area of reduced diffusion is depicted on the immediate postoperative MRI after resection of gliomas. On 1-month follow-up MRI, there is a clear focus of abnormal contrast enhancement along the resection margin corresponding to the area of the reduced diffusion on the postoperative MRI. This new enhancement, which can be easily misinterpreted as recurrent tumor, represents an area of brain injury with subsequent BBB disruption rather than recurrent tumor. Recent study shows that this enhancement within the area of diffusion abnormality invariably evolves into a gliotic cavity, as one would expect in any permanently injured brain, and does not represent tumor recurrence. ${ }^{19}$ Thus, it is imperative to evaluate any new enhancement within the first few months after surgery in the context of diffusion abnor- mality on the immediate postoperative MRI after tumor resection.

Diffusion tensor imaging is the latest application of diffusion-weighted MRI, in which white matter integrity can be depicted on a three-dimensional map. ${ }^{20}$ Although in its clinical infancy, diffusion tensor imaging and tractography are promising, noninvasive tools to study the white matter tracts and, with further development and validation, are likely to become an important and integral part of preoperative planning of brain tumors in the future.

One of the major pitfalls of diffusion-weighted imaging is related to the intrinsic sensitivity of the technique to lesions containing high magnetic susceptibility such as blood products, calcium or metal, and bone or air. The susceptibility artifact caused by the paramagnetic or ferromagnetic material can cause spurious signal changes on MR image that simulate pathologic process such as infarct or abscess and hence the interpretation of diffusion-weighted images must be done by concomitant re-
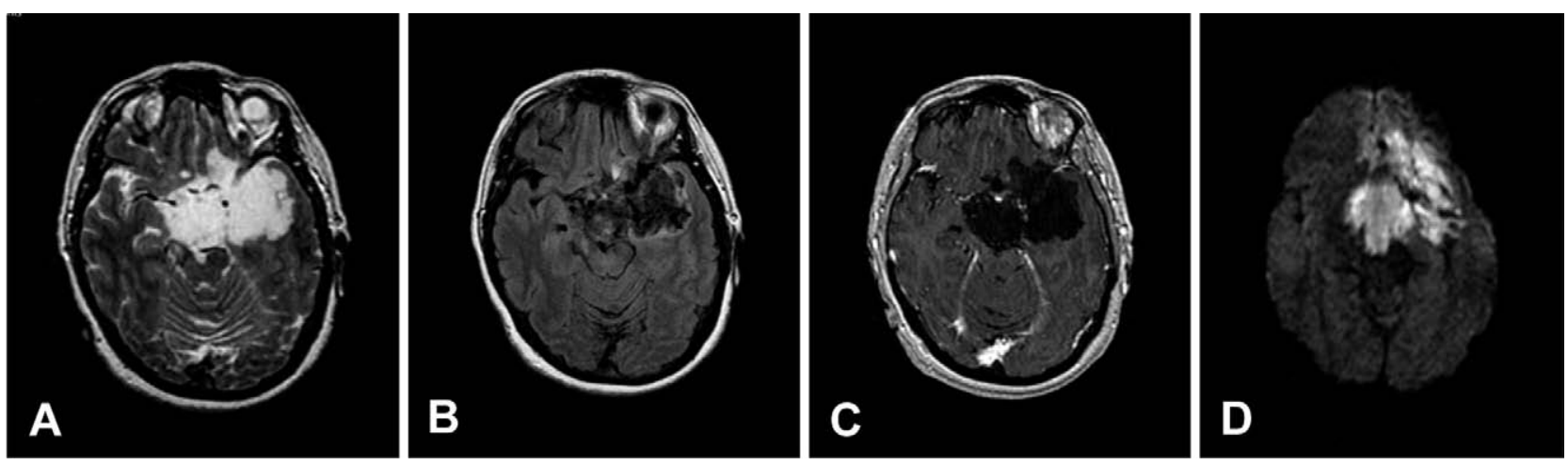

FIG. 5. A 45-year-old man with left middle fossa epidermoid. A: Axial $T_{2}$-weighted image shows a homogeneously bright lesion in the suprasellar and middle fossa region. B: Axial FLAIR image shows heterogeneously low signal within the mass. C: Axial contrastenhanced $\mathrm{T}_{1}$-weighted image shows no enhancement associated with the mass. D: Axial diffusion-weighted image demonstrates marked restricted diffusion, consistent with an epidermoid. 

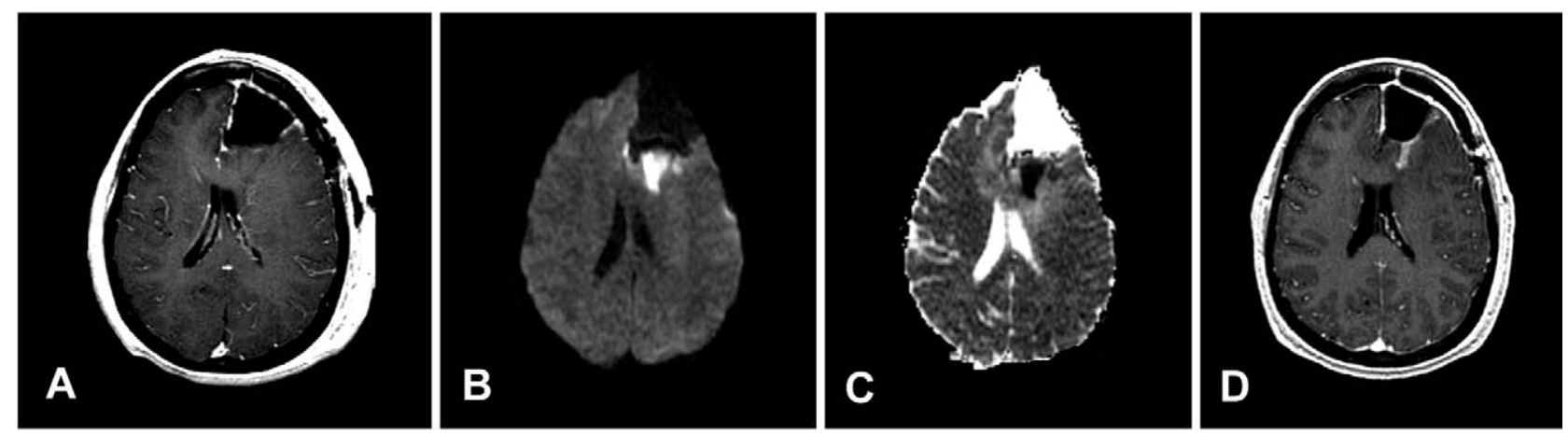

FIG. 6. A 47-year-old woman with anaplastic astrocytoma. A: Axial contrast-enhanced $\mathrm{T}_{1}$-weighted image immediately after surgery shows a left frontal resection cavity. B: Axial diffusion-weighted image shows a triangular shaped area of restricted diffusion at the posterior margin of the resection cavity. C: Apparent diffusion coefficient map confirms the restricted diffusion as evidenced by marked low signal. D: Axial contrast-enhanced $\mathrm{T}_{1}$-weighted image obtained 1 month after the immediate postoperative scan shows an area of enhancement that corresponds precisely to the area of restricted diffusion, consistent with postoperative changes rather than recurrent tumor.

view of anatomic MR images. This is particularly true in the immediate postoperative state, when there is usually a combination of blood products and surgical material within the surgical bed that can cause prominent susceptibility artifacts on diffusion-weighted imaging.

\section{Dynamic susceptibility-weighted contrast-enhanced perfusion MRI}

Dynamic susceptibility-weighted contrast-enhanced (DSC) perfusion MRI of the brain provides hemodynamic information that complements the anatomic information attainable with conventional MRI. Contrast-enhanced perfusion MRI methods exploit signal changes that accompany the passage of a paramagnetic contrast agent through the cerebrovascular system and that can be used to derive information on blood volume and flow. ${ }^{21-23}$ Dynamic perfusion MRI data analyzed using radiotracer kinetic theory yield quantitative estimates of cerebral blood volume (CBV) that reflect the underlying microvasculature and angiogenesis. This quick and robust technique is increasingly used as a research tool to evaluate and understand intracranial disease processes and as a clinical tool for diagnosis, management, and understanding of intracranial mass lesions, especially brain tumors. The vascularity of intracranial lesions, such as gliomas, ${ }^{24-26}$ cerebral lymphomas, ${ }^{27}$ and tumormimicking demyelinating lesions, ${ }^{28}$ has been assessed with perfusion MRI.

With the increasing number of applications of DSC MRI, it is important to understand the principles underlying the technique, and so this review surveys the essential underlying physics and methodology of DSC MRI. The clinical applications of CBV maps obtained from DSC MRI in the differential diagnosis of intracranial mass lesions are discussed, as are the pitfalls and limitations of the technique. Emphasis is on the clinical role of DSC MRI in providing insight into the underlying pathophysiology of cerebral microcirculation.

\section{Dynamic contrast-enhanced MRI}

Whereas DSC MRI is used to derive bulk vessel density, dynamic contrast-enhanced MRI (another type of contrast-enhanced perfusion MRI) is used primarily for estimation of vascular or endothelial permeability. Endothelial permeability of vessels in brain tumors provides valuable information about BBB integrity, vascular morphology, and the nature of neovascularization, as well as tumor pathophysiology and prognosis. ${ }^{29-31} \mathrm{Sev}$ eral recent studies have shown that quantitative estimates of microvascular permeability correlate with brain tumor grade. ${ }^{32-34}$ Current and potential clinical uses for a noninvasive method to characterize microvascular permeability in brain tumors include guiding a surgeon to the most malignant spot for biopsy, monitoring efficacy of chemotherapy or new treatments such as antiangiogenic drugs, manipulating the BBB for improved drug delivery, and differentiating radiation necrosis or postsurgical scar from recurring tumor.

The degree of endothelial permeability is typically represented by the endothelial permeability surface area product, $K^{\text {trans }}$, because MRI-derived $K^{\text {trans }}$ is a variable that can be compared across patient groups without correcting for physiologic variations between patients and for variations in imaging sequences and hardware. In addition to permeability, $K^{\text {trans }}$ also depends on several other factors, including vascular surface area and flow; $K^{\text {trans }}$ is therefore an indirect measure of physiologic parameters that vary with vascular density and angiogenic activity.

Analysis methods described by Tofts and Kermode ${ }^{35}$ have been widely used to determine $K^{\text {trans }}$ by MR using a steady-state contrast material-enhanced $\mathrm{T}_{1}$-weighted three-dimensional spoiled gradient-recalled acquisition sequence ( $\mathrm{ss}_{1}$ method) after the intravenous administration of gadopentetate dimeglumine. Although this approach affords high spatial resolution and is not subject 
to susceptibility artifact, both this method and a modified first-pass version require long imaging times and a complex postprocessing algorithm, and they may over- or underestimate $K^{\text {trans }}$.

A recently published method for quantifying $K^{\text {trans }}$ uses $\mathrm{T}_{2}{ }^{*}$-weighted gradient-echo echo-planar imaging during the first pass of gadolinium-based contrast agent $\left(\mathrm{fpT}_{2} *\right.$ method). ${ }^{36}$ In this approach, the dynamic data are converted to contrast agent concentration values in two compartments acquired during the first pass, and pharmacokinetic modeling is applied to $\mathrm{T}_{2}{ }^{*}$-weighted images of the first pass of a tracer bolus.

There are several theoretical differences between the $\mathrm{ssT}_{1}$ and the $\mathrm{fpT}_{2} *$ methods. First, whereas the contrast concentration versus time curve in the $\mathrm{ss}_{1}$ method fits a biexponential decay curve, the $\mathrm{fpT}_{2} *$ curve is gammavariate in shape. Based on the shape of these curves, the $K^{\text {trans }}$ value for normal brain is zero when derived by the $\mathrm{ssT}_{1}$ method but not zero (although very small or negligible) when determined using the $\mathrm{fpT}_{2} *$ method. Moreover, the rate of contrast movement from the intravascular to the extravascular space within a single voxel of tissue is assumed to be faster in the $\mathrm{fpT}_{2}{ }^{*}$ analysis, compared with the $\mathrm{ssT}_{1}$ analysis, and the intravascular contrast agent concentration is higher for $\mathrm{fpT}_{2} *$ imaging. By comparison with the $\mathrm{ssT}_{1}$ method, this newer technique is easier to implement, affords higher temporal resolution and broader section coverage, and allows simultaneous determination of relative cerebral blood volume (rCBV). Because $\mathrm{T}_{2} * \mathrm{rCBV}$ values are the current standard for perfusion imaging, we assessed the validity of the $\mathrm{T}_{2}{ }^{*} K^{\text {trans }}$ values by first performing a three-way comparison among $K^{\text {trans }}$ values derived from $\mathrm{ssT}_{1}$ and $\mathrm{fpT}_{2} *$ models and $\mathrm{T}_{2} * \mathrm{rCBV}$ values for correlation with glioma grade.

\section{CLINICAL APPLICATION OF PERFUSION MRI}

Vascular morphology and the degree of angiogenesis are important elements in evaluating different tumor types and in determining the biologic aggressiveness of intracranial neoplasms, especially gliomas. ${ }^{2,37}$ Tumor angiogenesis can be indirectly assessed using perfusion MRI-derived in vivo maps of CBV that depict the overall tumor vascularity. Perfusion MRI measurements of rCBV have been shown to correlate with both conventional angiographic assessments of tumor vascular density and histologic measurements of tumor neovascularization. Increased tumor vascularity is not synonymous with malignancy, however. Several intracranial neoplasms, especially those that are extra-axial, such as meningiomas or choroid plexus papillomas, can be highly vascular but nonetheless largely benign in biologic behavior.
In patients receiving antiangiogenic cancer therapies that directly attack tumor vessels, perfusion MRI is a noninvasive method to assess changes in the rCBV of the tumor during treatment and thus can be used to monitor the efficacy of therapy. Conventional MRI is limited by its nonspecificity and inability to differentiate between tumor recurrence and therapy-related necrosis. Findings of perfusion MRI have been shown to correlate better with clinical responses of patients undergoing antiangiogenic therapy. In addition, perfusion MRI (especially the dynamic contrast-enhanced approach) has become an important and integral part in the evaluation of antiangiogenic agents in neuro-oncology and will likely play an even greater role in the designing clinical trials and assessing treatment response in a whole new way by providing an objective, quantitative, and noninvasive imaging-based biomarker. ${ }^{38}$

\section{Gliomas}

Several studies have found a statistically significant correlation between the $\mathrm{rCBV}$ in a tumor and glioma grading. Studies have also shown statistically significant correlation between rCBV in the tumor and tumor vascularities determined using conventional catheter angiography. ${ }^{26}$ Because MRI can be used to quantitatively assess tumor vascularity, contrast-enhanced perfusion MRI can be used to measure CBV of the tumor, which reflects underlying tumor vascularity. Therefore, perfusion MRI-derived rCBV measurements can serve as noninvasive surrogate markers of tumor angiogenesis and malignancy. The implications of these findings are important, because vascular morphology is a critical parameter in primary high-grade gliomas in determining the potential for malignancy and for survival.

Low-grade astrocytomas have significantly lower mean rCBV than do anaplastic astrocytomas or glioblastomas. $^{24,25}$ Low-grade astrocytomas show little or no elevation in the CBV in the tumor, compared with the contralateral uninvolved brain. Anaplastic astrocytomas tend to have a higher rCBV than do low-grade astrocytomas, but lower rCBV than glioblastomas. The progressive increase in rCBV from low-grade to high-grade tumors is consistent with studies showing that microvascular density in low-grade astrocytomas is significantly lower than in anaplastic astrocytomas or glioblastomas, with glioblastomas being the most vascularized type of tumor. However, not only do the measurements of rCBV in different glioma grades overlap, but also rCBV measurements can and do vary considerably, because of the inherent extreme histologic heterogeneity of gliomas. Therefore, maps of rCBV of gliomas should not be interpreted without a concomitant evaluation of conventional MRI, which can provide other valuable information, such as the integrity of the BBB or the degree and characteristics of $\mathrm{T}_{2}$ abnormality. 

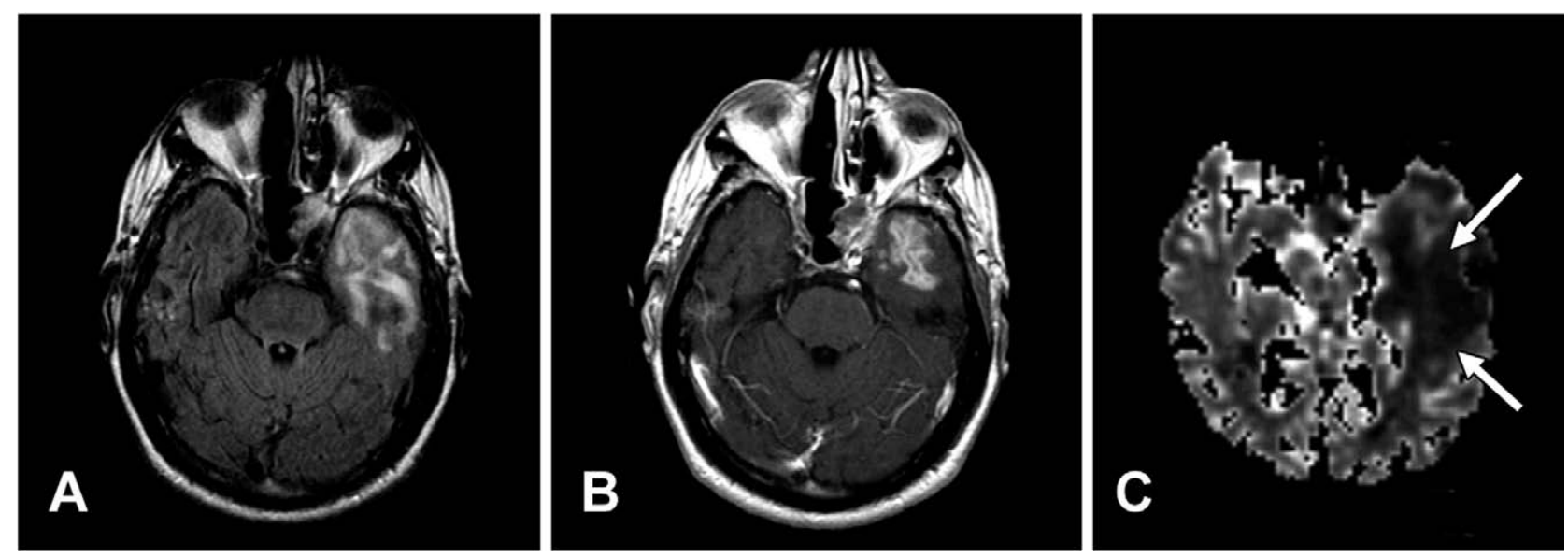

FIG. 7. A 58-year-old man with left temporal radiation necrosis with prior history of radiation therapy for clival chordoma. A: Axial FLAIR image shows a lesion with edema within the left temporal lobe. B: Axial contrast-enhanced $\mathrm{T}_{1}$-weighted images shows irregular enhancement of the left temporal lobe mass. C: Axial perfusion map from dynamic susceptibility-weighted imaging shows a large area of decreased blood volume (arrows) corresponding to the left temporal lobe mass. Extensive radiation necrosis was found at surgery.

Biopsy remains the definitive method for determining tumor type and grade; however, the well-known sampling error rate in biopsies of high-grade gliomas is caused in part by the extreme geographic heterogeneity within a single tumor. Ideally, the grading of gliomas should be based on histologic evaluation of tissue from the most malignant area in the tumor, but identifying this region can be difficult. In most biopsies, the imaging modality used for guidance is contrast-enhanced $\mathrm{T}_{1^{-}}$ weighted MRI or $\mathrm{CT},{ }^{39}$ which depict areas of $\mathrm{BBB}$ breakdown-but those areas may not correspond with the most malignant or most vascular portion of the tumor. Selecting a biopsy target on the basis of contrastenhanced $\mathrm{T}_{1}$-weighted MRI alone may be challenging. Maps of CBV can depict regions of increased vascularity that can serve as additional targets for stereotactic biopsy. At our institution, maps of rCBV are routinely used to select biopsy sites for both enhancing and nonenhancing tumors, and to help reduce sampling error and nondiagnostic biopsies. The rCBV map is particularly useful in patients with nonenhancing tumors, because the map can be used to locate the presumed site of increased tumor vascularity (i.e., the hot area).

Perfusion MRI has also been used as a prognostic tool to predict clinical outcome in patients with glioma. Law et al. ${ }^{40,41}$ have shown that CBV measurements of tumor derived from DSC MRI can predict time to progression or survival in patients with glioma and can be useful as an adjunct to histopathology in surgical and nonsurgical management of patients with low-grade glioma.

\section{Radiation necrosis and recurrent tumor}

Differentiation between radiation necrosis and recurrent tumor carries obvious therapeutic implications. Patients with recurrent tumors may benefit from undergoing a second operation and receiving adjuvant chemotherapy or targeted high-dose radiotherapy, whereas patients with radia- tion necrosis may be treated conservatively with steroids. Currently, the only definitive means of differentiating between radiation necrosis and recurrent tumor is histologic evaluation of tissue from biopsy or resection. However, surgical manipulation of areas of radiation necrosis can cause further damage to the adjacent brain parenchyma.

Delayed radiation necrosis is usually indistinguishable from recurrent tumor, both clinically and radiologically. Clinically, patients with either entity can present with progressive focal neurologic deficits and signs of increased intracranial pressure. On imaging, both entities can appear as a mass lesion with surrounding edema (FIGs. 7 and 8 . Conventional contrast-enhanced CT or MRI cannot be used to reliably distinguish radiation necrosis from recurrent tumor. Both processes can cause extensive edema and varying degrees of disruption in the BBB that result in mass effect and abnormal contrast enhancement, respectively. Pathologically, however, radiation necrosis and recurrent tumor are markedly dissimilar. Although the exact pathogenesis of delayed radiation necrosis remains obscure, a consistent pathologic feature is extensive endothelial injury and ultimate fibrinoid necrosis; in contrast, recurrent tumor is characterized by vascular proliferation. $^{42,43}$ The MRI-derived CBV mapping can reveal the pathologic differences in vascularity between therapyinduced necrosis and recurrent tumor and may help differentiate the two.

\section{Metastases}

Metastatic tumors, which make up almost $50 \%$ of all brain tumors, enter the central nervous system either hematogenously or by direct extension. Metastatic tumors induce neovascularization as they grow and expand. The newly formed capillaries resemble those of the primary systemic tumor, with fenestrated membranes and open endothelial junctions, all of which differ from 

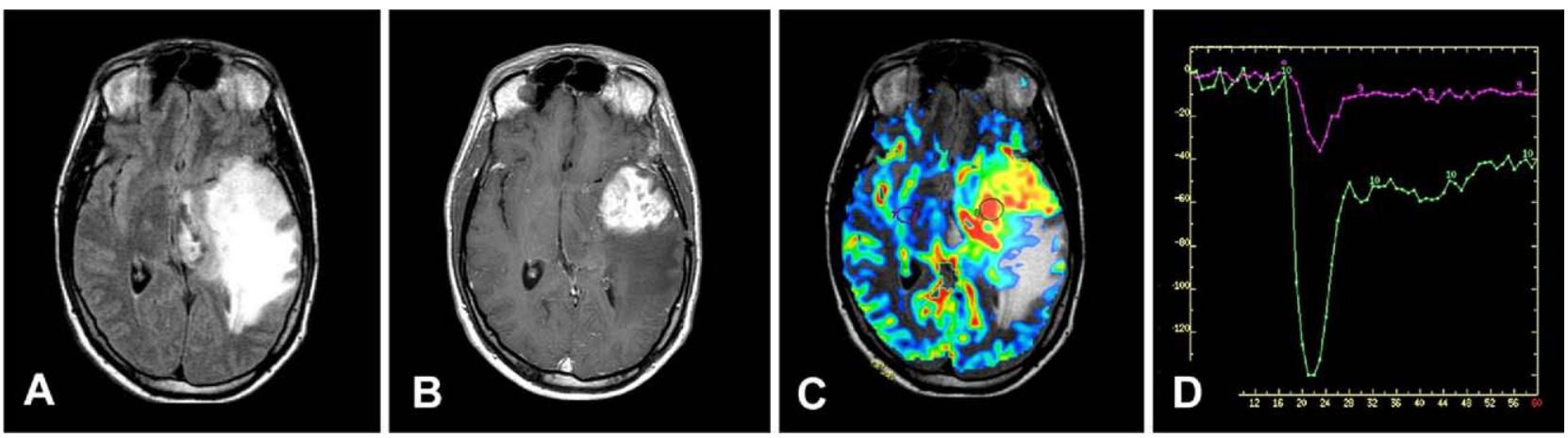

FIG. 8. A 52-year-old man with left temporal recurrent glioblastoma multiforme. A: Axial FLAIR image shows a lesion with edema within the left temporal lobe. B: Axial contrast-enhanced $\mathrm{T}_{1}$-weighted images shows irregular enhancement of the left temporal lobe mass. C: Axial color perfusion map from dynamic susceptibility-weighted imaging shows a large area of marked increased blood volume (orange and yellow areas), corresponding to the left temporal lobe mass. D: Dynamic susceptibility signal patterns within the tumor (green) and the contralateral white matter show marked difference in signal intensity drop between the two regions. Extensive tumor recurrence was found at surgery.

normal brain capillaries (which possess a well-developed BBB with tight junctions, a continuous basement membrane, and astrocytic foot processes). ${ }^{44}$ Intracranial metastases tend to be multiple lesions that enhance avidly on contrast-enhanced $\mathrm{T}_{1}$-weighted images with varying degrees of associated edema, and they are characteristically located near the junction of the gray and white matters. Hence, differentiating a metastatic brain lesion from a primary glioma usually presents no diagnostic dilemma. When a metastatic brain tumor presents as a solitary lesion, however, it can have an appearance similar to that of a glioma on both contrast-enhanced $\mathrm{T}_{1^{-}}$ weighted MR images and on rCBV maps.

Perfusion MRI may be useful in differentiating a solitary metastasis from a primary glioma on the basis of the difference in the measurements of peritumoral relative $\mathrm{CBV} .{ }^{45}$ This difference in the blood volume can be explained in part by the difference in the pathophysiology: of metastatic tumors. The peritumoral edema (defined as the area of hyperintensity on $\mathrm{T}_{2}$-weighted images in immediate contact with the enhancing tumor margin) is purely vasogenic edema that is caused by the increased interstitial water from leaky capillaries. ${ }^{46}$ In other words, in metastatic tumors, there is no histologic evidence of tumor beyond the outer contrast-enhancing margin of the tumor, and the peritumoral region represents the reaction of the surrounding intrinsically normal but edematous brain parenchyma. In high-grade gliomas, on the other hand, the peritumoral region represents a variable combination of vasogenic edema and tumor cells infiltrating along the perivascular spaces. Neoplastic cells can be found in some high-grade gliomas not only outside the contrast-enhancing margin but also well beyond the outer edge of the peritumoral zone visualized on $\mathrm{T}_{2^{-}}$ weighted MR images. ${ }^{39}$ By exploiting the pathophysiologic differences in the peritumoral region, perfusion MRI-derived blood volume measurements may help differentiate tumor infiltrated edema (in case of high-grade gliomas) from purely vasogenic edema (in case of metastasis).

\section{Meningiomas}

Meningiomas are highly vascular, extra-axial tumors that derive blood supply mostly from meningeal arteries with tumor capillaries that completely lack a BBB. Angiographically, meningiomas appear as hypervascular extra-axial masses that exhibit diffuse, homogeneous, and prolonged staining. Similarly, meningiomas are hypervascular on perfusion MRI. Because of the lack of a BBB within the tumor, the capillaries of meningioma are highly leaky and permeable. This phenomenon is apparent during the first-pass contrast agent bolus, when there is immediate contrast agent leakage without any substantial recovery of $\mathrm{T}_{2}{ }^{*}$ signal loss back to the baseline. Therefore, the perfusion MRI-derived rCBV measurements of meningiomas may be grossly overestimated or underestimated because of first-pass leakage-which essentially renders the intravascular compartmentalization of contrast agent impossible.

Similar to diffusion-weighted imaging, perfusion MRI is highly sensitive to susceptibility artifact due to echoplanar and $\mathrm{T}_{2}{ }^{*}$ effects of the technique. Any paramagnetic or ferromagnetic material can therefore cause severe artifact, particularly near the brain-bone-air interface near the middle cranial fossa or posterior fossa. Perfusion MRI of tumors in these locations is therefore intrinsically limited and challenging. In the immediate postoperative setting, perfusion MRI is often hampered by susceptibility artifact caused by blood products and surgical material.

\section{PROTON MRS}

\section{Basic physics}

Magnetic resonance spectroscopy (MRS) is a noninvasive MRI technique that produces metabolic spectra 
instead of generating anatomical images. There are several nuclei (e.g., ${ }^{1} \mathrm{H}$ proton, phosphorus, carbon, sodium, fluorine) that can be used for MRS, but proton (i.e., the hydrogen-1 nucleus) is the most commonly used, because of its abundance and high nuclear magnetic sensitivity. Proton MRS captures the biochemical signature of normal and diseased brain in vivo. Despite a large number of metabolites present in the brain, only freely mobile metabolites are detectable by MRS. Because water protons far outnumber the protons of other metabolites, it is critical to suppress water signal adequately, in order to detect the miniscule amount of important metabolites that are normally obscured by water proton signal.

The acquisition of MRS involves first defining the three-dimensional volume of interest to be studied. Localization of the volume of interest can be done by either single-voxel or chemical-shift techniques. In singlevoxel spectroscopy, a small region of brain (the usual minimum is $1 \mathrm{~cm}^{3}$ ) is interrogated to obtain metabolic information; it is a fast and easy technique, but limited by tissue coverage. Chemical-shift imaging, on the other hand, offers larger coverage and improved signal detection. For brain tumors, chemical-shift imaging is preferable due to its ability to provide metabolic information on a larger target area; however, this method requires a longer imaging time and complex data processing. Two essential steps involved in MRS acquisition involve sufficient water suppression and shimming the magnetic field (i.e., adjusting the resolution by optimizing the homogeneity of the magnetic field) to ensure homogeneity.

The major brain metabolites detected by proton MRS are NAA, choline, creatine, myoinositol, lipid, lactate, and glutamine, and glutamate. Each metabolite has corresponding proposed biochemical correlates: NAA is a marker of neuronal integrity; choline, of membrane turnover; creatine, an energetic marker; myoinositol, an astrocytic marker; lipid, a tissue destruction or necrosis marker; lactate, a hypoxia marker; and glutamine and glutamate, excitatory markers. Each metabolite is characterized by a specific resonance frequency with peak height, width, and area. Height or area under the peak can be calculated to yield a relative measure of the concentrations of protons.

\section{Clinical application of proton MRS}

Proton MRS has been shown to be useful in the evaluation of brain tumors and other mass lesions of the brain. Because actively dividing cells require membrane turnover, choline peaks tend to be high in brain tumors, whereas NAA peaks tend to be low, because of destruction of neurons. Several potential clinical application of proton MRS have been proposed, including guiding surgical brain biopsy and tumor grading. Although proton MRS has shown much promise as a helpful diagnostic tool to further characterize intracranial mass lesions, its contribution to outcome remains unknown. Potential clinical applications of proton MRS include image-guided surgical brain biopsy and glioma grading.

Image-guided surgical brain biopsy. Tissue heterogeneity is well known in brain tumors, and especially in gliomas, as is inherent sampling error associated with surgical biopsy. Conventional MRI is limited in its ability to guide biopsy sites to the most aggressive portion of the tumor, particular those without conventional contrast enhancement. Proton MRS has been used in guiding surgical biopsy to the area of high cellularity. ${ }^{47}$ Although proton MRS is unlikely to replace surgical biopsy for the diagnosis of brain tumor, it may offer advantages in selecting the appropriate target for biopsy. A three-dimensional proton MRS using chemical-shift imaging can capture metabolic information from a large portion of tumor. This may localize the most active or aggressive portion of tumor that can serve as a site for biopsy. Areas of high choline metabolites have been shown to correlate with high tumor proliferative index. It is important to recognize, however, that the minimum voxel size for MRS is $1 \mathrm{~cm}^{3}$, whereas a biopsy tissue specimen may be smaller than $1 \mathrm{~mm}^{3}$. This discrepancy in size must be kept in mind when choosing a biopsy site based on MRS and interpretation of biopsy result.

Glioma grading. Conventional MRI is limited in its ability to reliably grade gliomas prior to surgery. Contrast enhancement on postcontrast $\mathrm{T}_{1}$-weighted images is one of the most common methods for assessing glioma grade. As already noted, contrast enhancement is not synonymous with malignancy, and less aggressive tu-

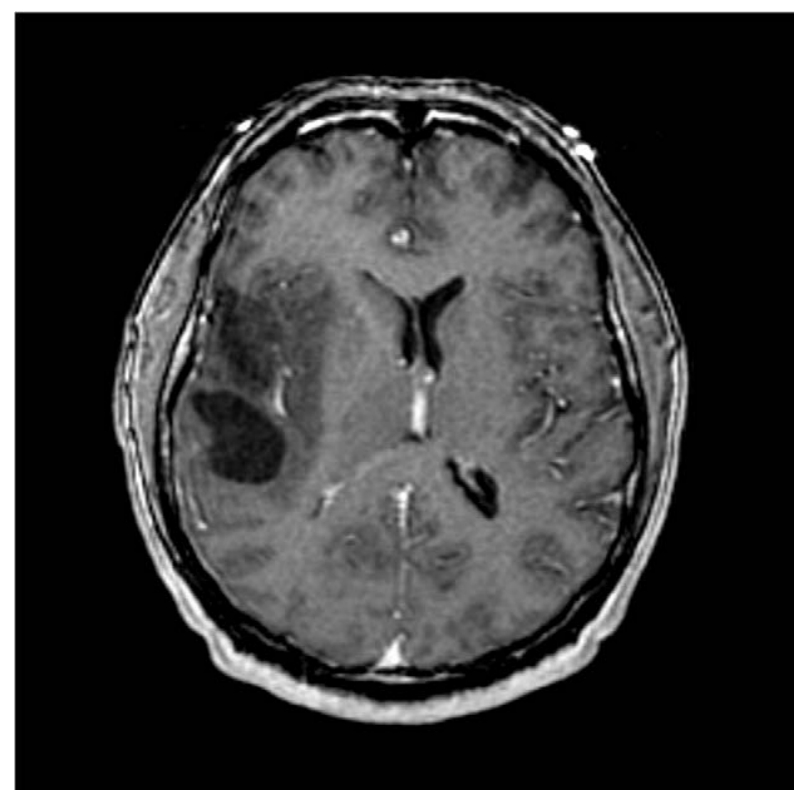

FIG. 9. A 38-year-old woman with right temporal glioblastoma multiforme. Axial contrast-enhanced $\mathrm{T}_{1}$-weighted image shows a nonenhancing, cystic appearing right temporal lobe mass, suggestive of a lower grade tumor. 
mors such as pilocytic astrocytomas and meningiomas often are avidly enhancing tumors. Grading based solely on conventional MRI is therefore unreliable. Similar to perfusion MRI, MRS is a promising noninvasive tool to assess tumor grade preoperatively. Conventional contrast-enhanced $\mathrm{T}_{1}$-weighted images may not be able to predict aggressive histology (FIG. 9), and the presence of lipid or lactate on MRS is highly suggestive of higher grade, malignant gliomas ${ }^{48}$ (FIG. 10).

Limitations. There are several important limitations associated with clinical application of proton MRS for brain tumor imaging. First, due to limitations in MRS voxel size, the entire tumor volume may not be interrogated and important areas of tumor may be missed. Second, there is no single MRS signature characteristic that correlates with tumor malignancy, and nonspecific spectral findings are not uncommon. Third, MRS data processing remains cumbersome, especially in case of multidimensional datasets, with offline workstation hardware and sophisticated software requirements. Further work is needed to improve MRS acquisition and data interpretation before this approach can be incorporated as part of routine clinical brain tumor imaging protocols.

\section{PITFALLS OF PHYSIOLOGICAL IMAGING}

Three important pitfalls are associated with the three aforementioned physiological MRI methods. First, because all three methods are based on echo-planar imaging technique, they are prone to susceptibility artifact and geometric distortion. The susceptibility artifact is most pronounced at the brain-bone-air interface, such as the anterior and middle cranial fossa or cerebellar hemisphere near the petrous apex. Brain tumors located in these regions may therefore not be suitable for physiological imaging. Second, there are other paramagnetic or ferromagnetic materials within the brain (e.g., blood products or calcium) that can simulate pathology on diffusion-weighted and perfusion MRI. As seen in FIG. 4, an intracranial parenchymal hematoma can show apparent reduced diffusion on DWI due to susceptibility effect caused by subacute and chronic blood products, methemoglobin and hemosiderin. Third, because of limited coverage of the brain in perfusion MRI and proton MRS not all of the brain tumor in question may be included in the imaging plane. This limited coverage is particularly of concern in large brain tumors or in brain tumors located near the cortex and skull.
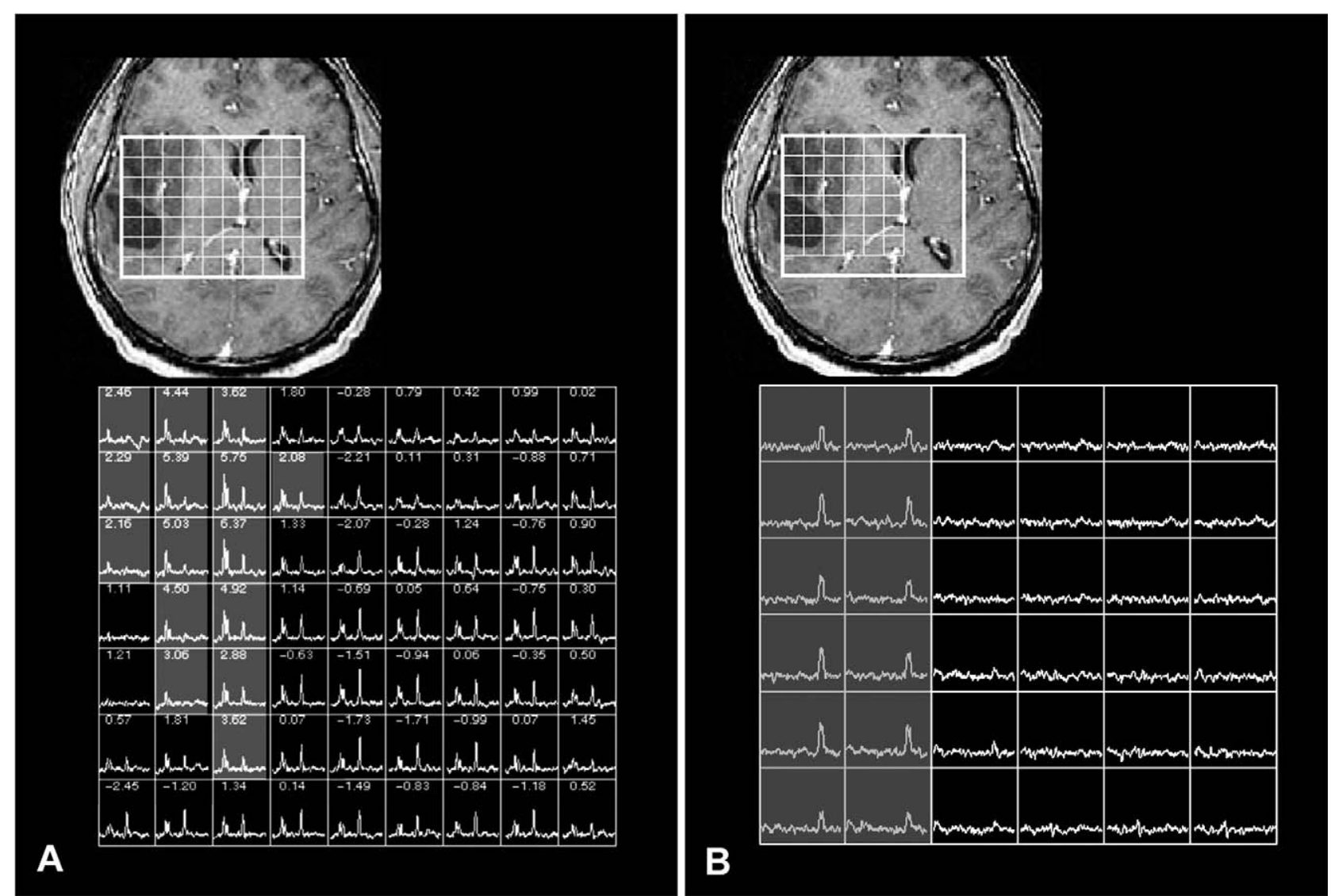

FIG. 10. Proton MRS study of a 38-year-old woman with right temporal glioblastoma multiforme (same patient as FIG. 9). A: Multivoxe MRS shows multiple voxels with abnormally high choline metabolites (gray shaded areas). B: Lactate-edited MRS shows multiple voxels with high lactate (shaded areas). Despite the less aggressive imaging feature on contrast-enhanced $\mathrm{T}_{1}$-weighted image, this mass was confirmed at surgery to be a glioblastoma multiforme. 


\section{CONCLUSIONS}

The current neuroimaging standard for brain tumor diagnosis and management is magnetic resonance imaging with intravenous administration of gadolinium-based contrast agent, which provides superb anatomical detail with multiplanar capability. With advances in imaging, image processing, and computer technology, however, physiology-based MRI methods are being developed and clinically applied to complement the morphologic findings from anatomical MRI with metabolic and functional information. Combined anatomic and physiologic MRI promises more comprehensive characterization of tumor and hence, a better understanding of tumor biology.

Diffusion-weighted MRI, contrast-enhanced perfusion MRI, and proton MRS imaging are prototypes of the physiology-based MRI approaches that can provide valuable information not readily discernable from anatomical MRI. No longer simply research tools, physiology-based MRI methods are rapidly become a part of routine brain tumor imaging protocols to improve diagnosis of intracranial mass lesions and management of patients with brain tumor. There are concerted efforts to combine all the anatomic and physiologic MRI techniques described in this review in a multiparametric, algorithmic fashion, in order to rapidly incorporate them into the clinical practice of neuroimaging and to benefit patients with brain tumor. ${ }^{49,50}$ Despite a potentially powerful role in advancing knowledge of brain tumor biology, physiologybased MRI methods still await much-needed validation and correlation with clinical outcome to determine whether (and if so, how) they may become a critical and indispensable part of clinical management of patients with brain tumor.

\section{REFERENCES}

1. Burger PC, Vogel FS. The brain: tumors. In: Burger PC, Vogel FS, editors. Surgical pathology of the central nervous system and its coverings. 2nd ed. New York: Wiley 1982;223-266.

2. Burger PC, Vogel FS, Green SB, Strike TA. Glioblastoma multiforme and anaplastic astrocytoma: pathologic criteria and prognostic implications. Cancer 1985;56:1106-1111.

3. Kleihues P, Sobin LH. World Health Organization classification of tumors. Cancer 2000;88:2887.

4. Kleihues P, Ohgaki H. Phenotype vs genotype in the evolution of astrocytic brain tumors. Toxicol Pathol 2000;28:164-170.

5. Ricci PE. Imaging of adult brain tumors. Neuroimaging Clin $\mathrm{N}$ Am 1999;9:651-669.

6. Felix R, Schorner W, Laniado M, et al. Brain tumors: MR imaging with gadolinium-DTPA. Radiology 1985;156:681-688.

7. Kates R, Atkinson D, Brant-Zawadzki M. Fluid-attenuated inversion recovery (FLAIR): clinical prospectus of current and future applications. Top Magn Reson Imaging 1996;8:389-396.

8. Ercan N, Gultekin S, Celik H, Tali TE, Oner YA, Erbas G. Diagnostic value of contrast-enhanced fluid-attenuated inversion recovery MR imaging of intracranial metastases. AJNR Am J Neuroradiol 2004;25:761-765.

9. Singer MB, Atlas SW, Drayer BP. Subarachnoid space disease: diagnosis with fluid-attenuated inversion-recovery MR imaging and comparison with gadolinium-enhanced spin-echo MR imaging-blinded reader study. Radiology 1998;208:417-422.
10. Maher EA, Furnari FB, Bachoo RM, et al. Malignant glioma: genetics and biology of a grave matter. Genes Dev 2001;15:13111333.

11. Edelman RR, Wielopolski P, Schmitt F. Echo-planar MR imaging. Radiology 1994;192:600-612.

12. Castillo M, Mukherji SK. Diffusion-weighted imaging in the evaluation of intracranial lesions. Semin Ultrasound CT MR 2000;21: 405-416.

13. Schaefer PW, Grant PE, Gonzalez RG. Diffusion-weighted MR imaging of the brain. Radiology 2000;217:331-345.

14. Holodny AI, Ollenschlager M. Diffusion imaging in brain tumors. Neuroimaging Clin N Am 2002;12:107-124, x.

15. Akai H, Mori H, Aoki S, et al. Diffusion tensor tractography of gliomatosis cerebri: fiber tracking through the tumor. J Comput Assist Tomogr 2005;29:127-129.

16. Chen SQ, Kang Z, Hu XQ, Hu B, Zou Y. Diffusion tensor imaging of the brain in patients with Alzheimer's disease and cerebrovascular lesions. J Zhejiang Univ Sci B 2007;8:242-247.

17. Mukherjee P, Berman JI, Chung SW, Hess CP, Henry RG. Diffusion tensor MR imaging and fiber tractography: theoretic underpinnings. AJNR Am J Neuroradiol 2008;29:632-641.

18. Nimsky C, Grummich P, Sorensen AG, Fahlbusch R, Ganslandt O. Visualization of the pyramidal tract in glioma surgery by integrating diffusion tensor imaging in functional neuronavigation. Zentralbl Neurochir 2005;66:133-141.

19. Smith JS, Cha S, Mayo MC, et al. Serial diffusion-weighted magnetic resonance imaging in cases of glioma: distinguishing tumor recurrence from postresection injury. J Neurosurg 2005;103:428438.

20. Ito R, Mori S, Melhem ER. Diffusion tensor brain imaging and tractography. Neuroimaging Clin N Am 2002;12:1-19.

21. Rosen BR, Belliveau JW, Vevea JM, Brady TJ. Perfusion imaging with NMR contrast agents. Magn Res Med 1990;14:249-265.

22. Weisskoff R, Belliveau J, Kwong K, Rosen B. Functional MR imaging of capillary hemodynamics. In: Potchen E, editor. Magnetic resonance angiography: concepts and applications. St. Louis: Mosby-Year Book, 1993:473-484.

23. Weisskoff RM, Rosen BR. Noninvasive determination of regional cerebral blood flow in rats using dynamic imaging with Gd(DTPA). Magn Reson Med 1992;25:211-212.

24. Aronen HJ, Gazit IE, Louis DN, et al. Cerebral blood volume maps of gliomas: comparison with tumor grade and histologic findings. Radiology 1994;191:41-51.

25. Knopp EA, Cha S, Johnson G, et al. Glial neoplasms: dynamic contrast-enhanced $\mathrm{T}_{2}{ }^{*}$-weighted MR imaging. Radiology 1999; 211:791-798

26. Sugahara T, Korogi Y, Kochi M, et al. Correlation of MR imagingdetermined cerebral blood volume maps with histologic and angiographic determination of vascularity of gliomas. AJR Am J Roentgenol 1998;171:1479-1486.

27. Sugahara T, Korogi Y, Shigematsu Y, et al. Perfusion-sensitive MRI of cerebral lymphomas: a preliminary report. J Comput Assist Tomogr 1999;23:232-237.

28. Cha S, Pierce S, Knopp EA, et al. Dynamic contrast-enhanced $\mathrm{T}_{2}$ *-weighted MR imaging of tumefactive demyelinating lesions. AJNR Am J Neuroradiol 2001;22:1109-1116.

29. Stewart PA, Hayakawa K, Farrell CL, Del Maestro RF. Quantitative study of microvessel ultrastructure in human peritumoral brain tissue: evidence for a blood-brain barrier defect. J Neurosurg 1987;67:697-705.

30. Uematsu H, Maeda M, Sadato N, et al. Vascular permeability: quantitative measurement with double-echo dynamic MR imaging: theory and clinical application. Radiology 2000;214:912-917.

31. Yang S, Law M, Zagzag D, et al. Dynamic contrast-enhanced perfusion MR imaging measurements of endothelial permeability: differentiation between atypical and typical meningiomas. AJNR Am J Neuroradiol 2003;24:1554-1559.

32. Provenzale JM, Wang GR, Brenner T, Petrella JR, Sorensen AG. Comparison of permeability in high-grade and low-grade brain tumors using dynamic susceptibility contrast MR imaging. AJR Am J Roentgenol 2002;178:711-716.

33. Roberts HC, Roberts TP, Brasch RC, Dillon WP. Quantitative measurement of microvascular permeability in human brain tu- 
mors achieved using dynamic contrast-enhanced MR imaging: correlation with histologic grade. AJNR Am J Neuroradiol 2000;21:891-899.

34. Roberts HC, Roberts TP, Ley S, Dillon WP, Brasch RC. Quantitative estimation of microvascular permeability in human brain tumors: correlation of dynamic Gd-DTPA-enhanced MR imaging with histopathologic grading. Acad Radiol 2002;9 Suppl 1:S151S155.

35. Tofts PS, Kermode AG. Measurement of the blood-brain barrier permeability and leakage space using dynamic MR imaging: 1. Fundamental concepts. Magn Reson Med 1991;17:357-367.

36. Johnson G, Wetzel SG, Cha S, Babb J, Tofts PS. Measuring blood volume and vascular transfer constant from dynamic, $\mathrm{T}_{2}{ }_{-}$ weighted contrast-enhanced MRI. Magn Reson Med 2004;51:961968.

37. Burger PC, Vollmer RT. Histologic factors of prognostic significance in the glioblastoma multiforme. Cancer 1980;46:11791186.

38. Batchelor TT, Sorensen AG, di Tomaso E, et al. AZD2171, a pan-VEGF receptor tyrosine kinase inhibitor, normalizes tumor vasculature and alleviates edema in glioblastoma patients. Cancer Cell 2007;11:83-95.

39. Kelly PJ, Daumas-Duport C, Scheithauer BE, Kall BA, Kispert DB. Stereotactic histologic correlations of computed tomography and magnetic resonance imaging defined abnormalities in patients with glial neoplasms. Mayo Clin Proc 1987;62:450-459.

40. Law M, Young R, Babb J, et al. Comparing perfusion metrics obtained from a single compartment versus pharmacokinetic modeling methods using dynamic susceptibility contrast-enhanced perfusion MR imaging with glioma grade. AJNR Am J Neuroradiol 2006;27:1975-1982.
41. Law M, Young RJ, Babb JS, et al. Gliomas: predicting time to progression or survival with cerebral blood volume measurements at dynamic susceptibility-weighted contrast-enhanced perfusion MR imaging. Radiology 2008;247:490-498.

42. Valk PE, Dillon WP. Radiation injury of the brain. AJNR Am J Neuroradiol 1991;12:45-62.

43. Ricci PE, Karis JP, Heiserman JE, et al. Differentiating recurrent tumor from radiation necrosis: time for re- evaluation of positron emission tomography? AJNR Am J Neuroradiol 1998;19:407413.

44. Vajkoczy P, Menger MD. Vascular microenvironment in gliomas. J Neurooncol 2000;50:99-108.

45. Law M, Cha S, Knopp EA, et al. High-grade gliomas and solitary metastases: differentiation by using perfusion and proton spectroscopic MR imaging. Radiology 2002;222:715-721.

46. Machein MR, Plate KH. VEGF in brain tumors. J Neurooncol 2000;50:109-120.

47. Burtscher IM, Skagerberg G, Geijer B, et al. Proton MR spectroscopy and preoperative diagnostic accuracy: an evaluation of intracranial mass lesions characterized by stereotactic biopsy findings. AJNR Am J Neuroradiol 2000;21:84-93.

48. Li X, Lu Y, Pirzkall A, McKnight T, Nelson SJ. Analysis of the spatial characteristics of metabolic abnormalities in newly diagnosed glioma patients. J Magn Reson Imaging 2002;16:229-237.

49. Al-Okaili RN, Krejza J, Wang S, Woo JH, Melhem ER. Advanced MR imaging techniques in the diagnosis of intraaxial brain tumors in adults. Radiographics 2006;26 Suppl 1:S173-S189.

50. Al-Okaili RN, Krejza J, Woo JH, et al. Intraaxial brain masses: MR imaging-based diagnostic strategy_initial experience. Radiology 2007;243:539-550. 